\title{
A Survey of the Proportion of Classes in Undergraduate Engineering Curricula that Include Labs
}

\section{Eleanor Byrnes, Harvey Mudd College}

Ellie Byrnes is a student at Harvey Mudd College, currently pursuing a BS in Mathematics. Ellie has an interest in doing work in STEM education and expects to graduate from in May of 2021.

Yaqub Alam Mahsud, Harvey Mudd College

Spencer Rosen, Harvey Mudd College

Spencer Rosen is a student at Harvey Mudd College pursuing a BS in Engineering with an emphasis on Electrical and Computer Engineering. He expects to graduate in May of 2020.

\section{Matthew Spencer, Harvey Mudd College}

Matthew Spencer is an assistant professor at Harvey Mudd College. His research interests include experiential and hands-on learning, and integrating mechanical, chemical and quantum devices into circuits and communication links. 


\section{A Survey of the Proportion of Classes in Undergraduate Engineering Curricula that Include Labs}

Abstract -- This research paper describes the results of a systematic review of engineering course catalogs and program requirements that sought to answer the question, "How many laboratory classes does an engineering student take?". This study is motivated by two observations: first, literature suggests that laboratories have historically served a critical role in the education of engineering students, but second, accreditation boards do not list requirements for the number of laboratories that engineers must take, instead offering the requirement a program provides "experimental experience appropriate to the program." This study provides a quantitative summary of how different programs interpret that charge, and that summary can be used as a benchmark for programs to evaluate their own laboratory offerings.

Naturally, the number of laboratory classes a student takes could be influenced by many factors, including programmatic resources and the requirements of different engineering sub-disciplines. Accordingly, catalogs and program requirements were collected from several engineering disciplines and from the top and bottom of college rankings. The number of lab and non-lab classes in each program were compared in a statistical linear model that accounted for the rank of the program and the engineering sub-discipline.

Results show that there are significant differences between the distributions of number of labs in electrical and mechanical engineering curricula, but that there are not significant differences based on the ranking of the schools. $50.4 \%$ of the required electrical engineering classes in an undergraduate electrical engineering program contain hardware-based laboratories, and 39.1\% of mechanical engineering courses require laboratories. The high proportion of laboratory classes in curricula reaffirms the importance of research into the pedagogy of laboratory design.

\section{Introduction}

Engineering is a discipline that requires interaction with the physical world, so it is of little surprise that there is broad consensus among engineering educators that laboratory classes are an important part of engineering curriculums [1]. However, the Accreditation Board for Engineering and Technology (ABET) doesn't specify how many laboratory classes should be in an engineering program, instead offering the guideline that laboratory experiences should be "appropriate to the program" [2]. Literature is also quiet on the question of "how many engineering laboratory classes does an undergraduate engineer take?" 
This study tried to answer that question by performing a systematic review of publicly available course catalogs to count the number of laboratories required of engineering students in different engineering programs. Naturally, the faculty in an engineering program might make decisions about how many laboratories to require based on many factors. This study focuses on two such factors: engineering discipline (e.g.: mechanical engineering is a discipline), and a school's ranking in a national college ranking website [3], which was used to represent the financial resources available to the program. The study considered two disciplines -- electrical engineering (EE) and mechanical engineering (ME) -- and two different clusters of school rankings -- highly and poorly ranked schools. This resulted in four categories of programs to analyze top EE, bottom EE, top ME and bottom ME.

While all schools in the dataset evaluated in this study reported credit hours using the Carnegie Unit, which corresponds to 3 hours of work per week, a statistically significant number of schools did not report credit hours for the laboratory component of classes. For example, at one school, Fluid Mechanics was listed as a three credit course while Fluid Mechanics Laboratory was listed as a zero credit course, despite being a co-requisite. This ambiguity in credit reporting is why the number of laboratory courses is used as a metric in this study instead of the number of credit hours.

A total of 271 sets of course catalogs and program requirements were reviewed to evaluate if they were suitable for the study, and the best thirty from each category were retained for analysis. Class descriptions were extracted from these documents, and these descriptions were categorized into multiple categories reflecting the many types of laboratory experiences students can have: no laboratory component, traditional in-lab hardware experiences, software-based laboratories [4], take home lab kits [5], mixed studio-lecture courses [6], or other laboratory experiences.

The hypothesis of this study was that both engineering discipline and school rank would drive significant differences in the number of laboratories a program offered because EE and ME have very different capital requirements for laboratory classes, and resources are one explicit aspect of school rankings. The hypothesis was assessed by building a statistical linear model and running an ANOVA on the proportion of laboratories uncovered by systematic review in each of the four categories of programs discussed above.

This process is described in the following sections: Section 2 describes the methods used for data collection and categorization, Section 3 describes the results of the analysis and Section 4 draws conclusions from the results and suggests avenues for future work. 


\section{Methods}

Three steps were required to gather the database used for this study: evaluating programs to determine if they contained viable classes, systematically selecting programs to review and categorizing the classes in reviewed programs based on the type of laboratory experience offered. This section discusses each of those steps.

\subsection{Evaluating Catalogs to Determine if They Contain Viable Classes}

An engineering program is often composed of requirements derived from multiple sources. For instance, a student might be required to complete general education requirements, a set of required engineering classes, and some elective classes of his/her choosing. Of these three categories, only the required engineering classes are reflective of courses required of engineers by an engineering program. General education requirements could be imposed by a wide variety of departments, so laboratories in general education requirements aren't guaranteed to be engineering laboratories. Similarly, there are no guarantees that any particular elective includes laboratories. Therefore, the first step of reviewing a program is examining the program requirements and catalog to determine how many general education, engineering requirements and electives are required of students. This classification is referred to as requirement type.

\subsection{Selecting Catalogs for Review}

After the courses in a program have been categorized by requirement type, a set of inclusion/exclusion criteria was applied to guarantee that programs in the review were suitable for statistical analysis. The criteria were:

1. EXCLUDE IF: The program does not provide a syllabus with course descriptions and requirements for the engineering discipline being considered.

2. EXCLUDE IF: Course descriptions are absent to a degree that it is impossible to determine if a course contains a laboratory experience.

3. EXCLUDE IF: Engineering general education requirements are not separated from engineering program requirements in the publicly available program descriptions.

4. EXCLUDE IF: The program is more than $40 \%$ electives.

5. INCLUDE IN TOP CATEGORY: The thirty top-most ranked programs in [3] of the engineering discipline being considered that were not excluded from the study.

6. INCLUDE IN BOTTOM CATEGORY: The thirty bottom-most ranked programs in [3] of the engineering discipline being considered that were not excluded from the study.

7. INCLUDE IN EE CATEGORY: If the engineering discipline being considered is electrical engineering

8. INCLUDE IN ME CATEGORY: If the engineering discipline being considered is mechanical engineering. 
Note that the phrasing of these criteria allows different sets of schools in each engineering subdiscipline.

Many of these requirements are self-explanatory -- e.g.: it's impossible to categorize courses if course descriptions are missing -- but the exclusion of programs that are $40 \%$ or more electives deserves explanation. In courses that are mostly electives, the proportion of laboratory classes that a student takes could vary widely depending on the classes the student selects. Moreover, the number of engineering requirements in these programs is very small, so even one required engineering lab can dramatically affect the proportion of labs in these programs. These two effects make highly elective programs similar to programs with missing course descriptions: it's impossible to make a good guess of how many courses contain laboratory components.

Using [3] as a source of ratings for inclusion in the top and bottom categories instead of other rankings -- [7] for example -- is a potentially controversial choice, but analysis suggests it doesn't matter much. Figure 1 shows a scatter plot comparing rankings in [3] and [7] for all programs included in the study, and it shows that highly rated schools and poorly rated schools form correlated clusters in the upper right and lower left. Because this analysis doesn't use a program's exact rank, rather binning programs into "high" or "low" ranked categories, few (14 of 120) schools would differ if the other list were used. Trying to leverage this correlation to get more accurate school rankings motivated the choice to use 30 programs in each category.

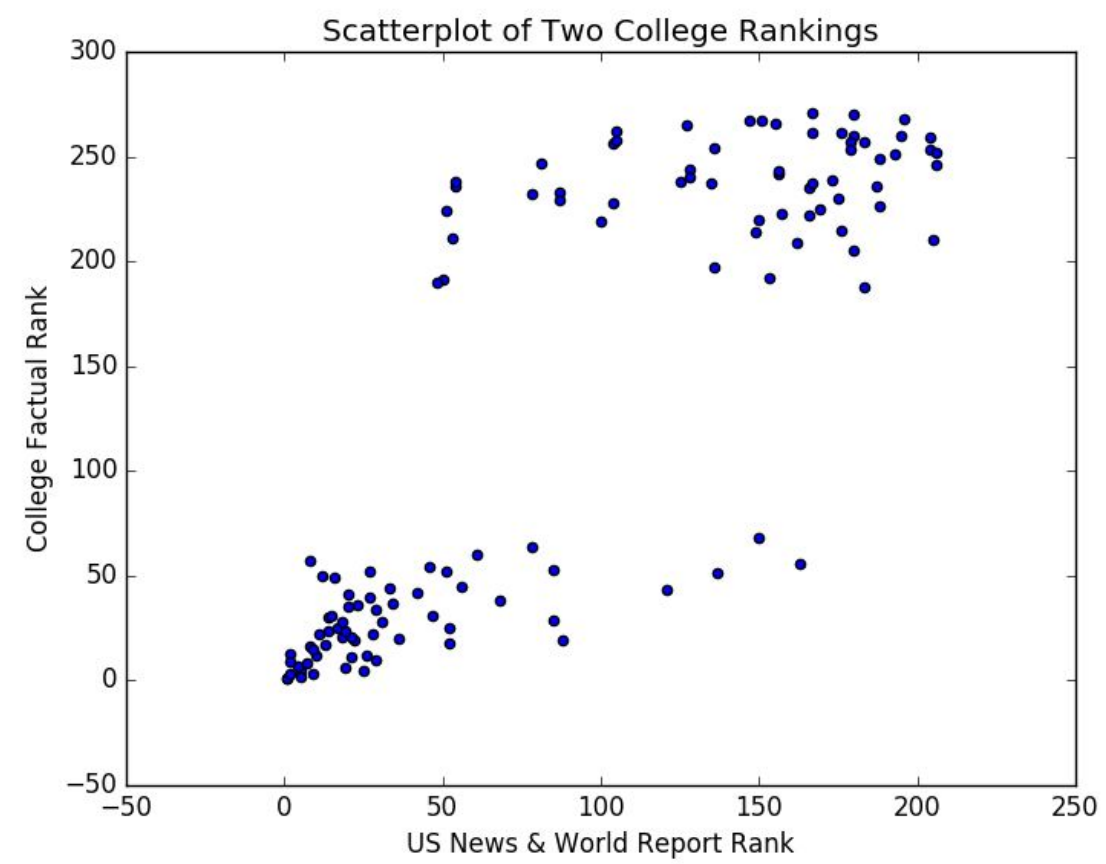

Figure 1: Scatter plot showing correlation between high and low rated schools in [3] and [7] 


\subsection{Categorizing classes}

Course descriptions were gathered from the course catalogs of included programs, and the descriptions were classified as laboratory classes that used hardware, laboratory classes that exclusively used software, or classes that had no laboratory component. The categories are described in greater detail below:

- Hardware laboratories -- laboratories in which students were asked to interact with an external piece of equipment or measure a natural phenomenon. This category included take-home lab kits and mixed studio-lectures.

- Software-only laboratories -- laboratories that consisted entirely of simulation or programming. Remote laboratories, where students interacted with a software interface to real equipment, were included in this category, as were finite element / simulation based labs.

- No laboratories -- no laboratory experiences.

A second reader evaluated a subset of the catalog descriptions to verify the validity of the codes. The inter-rater reliability of these evaluations was $88 \%$ (by Cohen's Kappa).

Readers reported that the features which indicated a course included a laboratory component included the following: use of the words "lab," "prototype," "build," or "measure" in the course description; the presence of a co-required course designated as a laboratory section; or significant discussion of implementing or measuring physical systems. Features indicating software laboratories included descriptions of simulations; the words "remote laboratory" or "web-based laboratory"; or the presence of a co-requisite computer laboratory section.

\section{Results}

The proportion of required engineering courses which contained laboratories was calculated for each program. A statistical linear model was constructed to predict the proportion assuming it was influenced by the type of program (ME/EE), the ranking cluster the program belonged to (high/low), an offset and noise. This model was used in an ANOVA test, and Table 1 summarizes the model and ANOVA findings.

The table shows that the engineering discipline has a high $\mathrm{F}$ score and low p score, suggesting that it has significant predictive power. The intercept is also significantly predictive. However, the table also suggests that the rank of programs has little predictive power: the Top/Bot code has a high p score and a low F score. 
Table 1: Statistical Linear Model

\begin{tabular}{|l|c|c|c|c|c|c|}
\hline & Sum of Squares & Mean Squares & Coefficient & Coeff. Variance & $\mathrm{p}$ & $\mathrm{F}$ \\
\hline EE/ME & 0.38 & 0.38 & -0.11 & 0.03 & 0.00 & 19.91 \\
\hline Top/Bot & 0.00 & 0.04 & 0.01 & 0.03 & 0.62 & 0.24 \\
\hline Intercept & N/A & N/A & 0.50 & 0.02 & N/A & N/A \\
\hline Residual & 2.22 & 0.02 & N/A & N/A & N/A & N/A \\
\hline
\end{tabular}

The distribution of proportions of laboratory courses for each of the four categories are pictured in Figure 2. EE distributions differ from ME, but top and bottom distributions for both EE and ME overlap substantially, which corroborates the statistical linear model. A t-test confirms this suspicion: the mean proportion of laboratory classes in EE and ME programs was significantly different ( $\mathrm{p}=1.7 \mathrm{e}-5)$. On average, $50.4 \%$ of classes required in an EE program were labs while $39.1 \%$ of classes required in an ME program were labs.

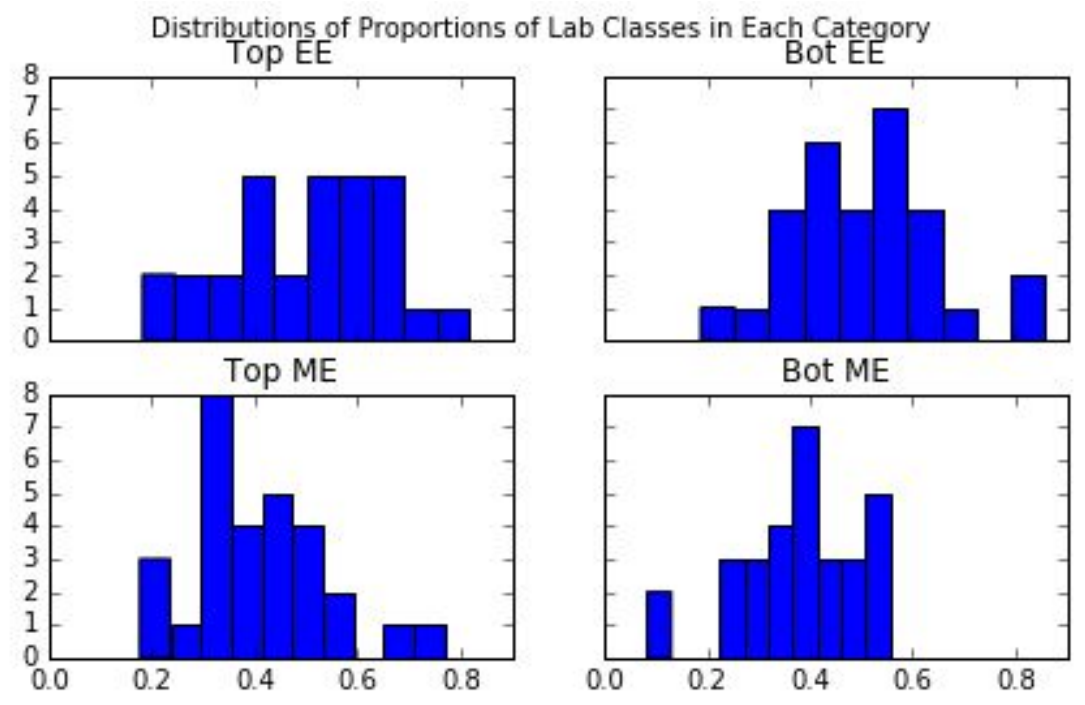

Figure 2: Distributions of the proportion of required classes that have hardware laboratory classes in each category used in this analysis.

These results confirm the hypothesis that engineering disciplines have different laboratory requirements, which could be driven by the cost of laboratories, different amounts of faculty labor required to maintain laboratories or different values in the disciplines. This result falsifies the hypothesis that program ranking is a useful indicator of the number of laboratories a program 
offers. The large proportions of classes which have laboratories in all types of programs suggest that engineering programs place high value on laboratory experiences, which corroborates [1].

\section{Future Work and Conclusions}

There are a number of obvious avenues to extend this work. First, the causal factors that influence how many labs programs opt to offer are still unclear, so there should be more work on determining those factors. Second, school rank is a weak proxy for program resources and it could be replaced by individual variables like number of faculty, number of staff and operating budget. Third, it would be helpful to review full syllabi for the courses being evaluated and to confirm the laboratory evaluations with staff or chairs from the surveyed programs. Finally, the survey should be expanded to cover more ground; chemical, biomedical and civil engineering have even higher capital requirements than mechanical engineering, so analyzing programs from these disciplines would expand the dataset in interesting directions.

However, the top line conclusions of this study -- engineering disciplines have different proportions of lab classes, but regardless of rank, EE programs include laboratories in $50 \%$ of required courses and ME programs include laboratories in $40 \%$ of their required courses -- are powerful indicators that laboratories are an important part of engineering education. Improving the teaching of laboratories has the potential to improve almost half of the classes that engineers take, so further research on the subject of engineering laboratory pedagogy is important work.

\section{REFERENCES}

[1] L. D. Feisel and A. J. Rosa, "The Role of the Laboratory in Undergraduate Engineering Education," J. Eng. Educ., vol. 94, no. 1, pp. 121-130, Jan. 2005, doi: 10.1002/j.2168-9830.2005.tb00833.x.

[2] "Criteria for Accrediting Engineering Programs, 2020 - $2021 \mid$ ABET." [Online]. Available: https://www.abet.org/accreditation/accreditation-criteria/criteria-for-accrediting-engineering-program s-2020-2021/. [Accessed: 30-Jan-2020].

[3] “College Factual," College Factual, 20-Feb-2013. [Online]. Available: https://www.collegefactual.com/. [Accessed: 31-Jan-2020].

[4] A. Yazidi, H. Henao, G.-A. Capolino, F. Betin, and F. Filippetti, "A Web-Based Remote Laboratory for Monitoring and Diagnosis of AC Electrical Machines," IEEE Trans. Ind. Electron., vol. 58, no. 10, pp. 4950-4959, Oct. 2011, doi: 10.1109/TIE.2011.2109331.

[5] R. M. Reck and R. S. Sreenivas, "Developing a new affordable DC motor laboratory kit for an existing undergraduate controls course," in 2015 American Control Conference (ACC), 2015, pp. 2801-2806, doi: 10.1109/ACC.2015.7171159.

[6] D. Millard, F. Berry, and M. Chouikha, "Improving Student Intuition Via Rensselaer's New Mobile Studio Pedagogy," presented at the 2007 Annual Conference \& Exposition, 2007, pp. 12.862.1-12.862.12.

[7] “The Best Colleges in America, Ranked." [Online]. Available: https://www.usnews.com/best-colleges. [Accessed: 31-Jan-2020]. 